\title{
Integrative omics analysis. A study based on Plasmodium falciparum mRNA and protein data
}

\author{
Oana A Tomescu, ${ }^{1,2}$, Diethard Mattanovich ${ }^{3,4}$, Gerhard G Thallinger ${ }^{1,2,5^{*}}$ \\ From High-Throughput Omics and Data Integration Workshop \\ Barcelona, Spain. 13-15 February 2013
}

\begin{abstract}
Background: Technological improvements have shifted the focus from data generation to data analysis. The availability of large amounts of data from transcriptomics, protemics and metabolomics experiments raise new questions concerning suitable integrative analysis methods. We compare three integrative analysis techniques (co-inertia analysis, generalized singular value decomposition and integrative biclustering) by applying them to gene and protein abundance data from the six life cycle stages of Plasmodium falciparum. Co-inertia analysis is an analysis method used to visualize and explore gene and protein data. The generalized singular value decomposition has shown its potential in the analysis of two transcriptome data sets. Integrative Biclustering applies biclustering to gene and protein data.

Results: Using CIA, we visualize the six life cycle stages of Plasmodium falciparum, as well as GO terms in a 2D plane and interpret the spatial configuration. With GSVD, we decompose the transcriptomic and proteomic data sets into matrices with biologically meaningful interpretations and explore the processes captured by the data sets. IBC identifies groups of genes, proteins, GO Terms and life cycle stages of Plasmodium falciparum. We show method-specific results as well as a network view of the life cycle stages based on the results common to all three methods. Additionally, by combining the results of the three methods, we create a three-fold validated network of life cycle stage specific GO terms: Sporozoites are associated with transcription and transport; merozoites with entry into host cell as well as biosynthetic and metabolic processes; rings with oxidation-reduction processes; trophozoites with glycolysis and energy production; schizonts with antigenic variation and immune response; gametocyctes with DNA packaging and mitochondrial transport. Furthermore, the network connectivity underlines the separation of the intraerythrocytic cycle from the gametocyte and sporozoite stages.

Conclusion: Using integrative analysis techniques, we can integrate knowledge from different levels and obtain a wider view of the system under study. The overlap between method-specific and common results is considerable, even if the basic mathematical assumptions are very different. The three-fold validated network of life cycle stage characteristics of Plasmodium falciparum could identify a large amount of the known associations from literature in only one study.
\end{abstract}

\section{Background}

Continuous technological improvements facilitate the availability of large amounts of omics data, resulting from the simultaneous characterization on different levels (genome, transcriptome, proteome and metabolome) of an organism or an experimental condition. Regulatory

\footnotetext{
* Correspondence: gerhard.thallinger@tugraz.at

'Core Facility Bioinformatics, Austrian Centre of Industrial Biotechnology, Graz, Austria

Full list of author information is available at the end of the article
}

mechanisms captured in this way provide a complex multi-level view of the system under study. In order to exploit the measured data to the maximum, one has to integrate all available data sets into a single analysis framework. Methods that apply analysis techniques simultaneously to more than one data set are called integrative analysis methods. The data sets can characterize one organism on different levels [1], or they can be measured on the same omics level but on different organisms/platforms $[2,3]$. Here we focus on the first scenario. 
Integrative analysis methods provide a deeper understanding of the system under study through the meaningful combination of multi-level omics data. The integrated omics data differ from study to study. There are studies that integrate, for example, gene expression and methylation data [4], somatic mutations, copy number and gene expression data [5], chromatin maps and gene expression profiles [6], genotypic variation at DNA level and gene expression data [7], CHIP-seq and RNA-seq data [8], transcriptomics and proteomics data $[1,9,10]$. In this study we apply integrative analysis to transcriptomics and proteomics data.

With transcriptomic and proteomic data, most analysis techniques are based on the direct correlation between transcripts and proteins. Cox and colleagues [10] present different approaches based on correlation and clustering. Other correlation-based studies have also been performed in [9,11-16]. Statistical methods based on correlations are presented in $[17,18]$. The premise of a direct correlation between transcripts and proteins is not valid in eucaryotic organisms, due to post-transcriptional and post-translational regulation $[1,19]$. Other approaches are based on network analysis $[20,21]$ and statistical methods such as analysis of variation, clustering and gene set enrichment [22-24]. Piruzian et al. [25] revealed similarities in regulation at transcriptomic and proteomic levels and identified potential key transcription factors and new signaling pathways for psoriasis using a network based approach, which employed overconnection analysis, hidden node analysis and rank aggregation. Perco et al. [26] integrated transcriptomics and proteomics on the level of protein interaction networks. They started with the modest overlap between the data sets, which increased substantially on the level of protein interaction networks and in this way, amplified the joint functional interpretation of the omics data sets. In a study by Hahne and colleagues [22] analysis of variation, k-means clustering and functional annotation were applied to transcriptome and proteome data from salt-stressed B. subtilis cells. They showed a well-coordinated induction of gene expression and changes of the protein levels as the result of a severe salt shock. Verhoef et al. characterized the changes associated with $\rho$-hydroxybenzoate production in the engineered $P$. putida strain S12, integrating genes and proteins as well as cluster and pathway analysis [23]. In [24], Takemasa et al. applied gene ontology analysis (GO) to transcriptome and proteome data from human colorectal cancer samples, which led to a better understanding of functional inference at the physiological level and to potential drug targets. Other integrative approaches can be found in [27-29] for omics data in general and in [19] for transcriptome and proteome data in particular.

In this article, we focus on the comparison of three integrative analysis techniques of mRNA and protein abundance data. We selected methods meeting the following criteria: (i) they are based on a clear mathematical formulation, (ii) they are as different as possible from one another and, the most important argument, (iii) they allow the analysis of all measured data (not limited to pairs of genes and proteins). Based on these criteria we have chosen: Co-inertia analysis (CIA), which is an integrative analysis method used to visualize and explore gene and protein data $[1,30]$, Generalized singular value decomposition (GSVD), which has shown its potential in the analysis of two transcriptome data sets [3] and Integrative biclustering (IBC), which applies biclustering to gene and protein data [31].

We compare CIA, GSVD and IBC by applying them to mRNA and protein abundance data from a study of Plasmodium falciparum, [9], the parasite causing malaria in humans. The data in this study was gathered from samples for the six life cycle stages: merozoite, ring, trophozoite, schizont, gametocyte and sporozoite. For the comparison, we add additional information in the form of $\mathrm{GO}$ [32] terms for biological processes.

Using CIA, we visualize the six life cycle stages and GO terms in a 2D plane and interpret the spatial configuration. With GSVD, we decompose the data sets into matrices with biologically meaningful interpretations and explore the processes captured by the data sets. IBC identifies groups of genes, proteins, GO terms and life cycle stages revealing functional modules of $P$. falciparum.

We compare the results of the three integrative analysis methods based on the association of GO terms to the six life cycle stages and show common as well as methodspecific results. The common results are presented in form of a three-fold validated network view of the biological processes activated in each life cycle stage. To the best of our knowledge such a complete, GO terms based, characterization of $P$. falciparum was not published before.

\section{Methods \\ Data set}

We analyse a publicly available data set containing mRNA and protein abundance data from the six life cycle stages of $P$. falciparum $[9,33]$. Microarray [33] and proteomic analyses [9] were carried out on P. falciparum clone 3D7. Gene expression levels were measured with a custom oligonucleotide array and computed with the match-only integral algorithm (MOID). Proteins were detected by multidimensional protein identification technology (MudPIT), and protein abundance was estimated by the number of MS/MS spectra identified per protein. In total, 4294 genes and 2903 proteins were measured in all six life cycle stages. For the analysis, we created a matrix for each data set where the genes and proteins are represented as rows and the life cycle stages as columns. 
Additionally, GO [32] information on biological processes in $P$. falciparum were employed. We used the $\mathrm{R}$ [34] packages org.Pf.plasmo.db [35] and GO.db [36], which provide $P$. falciparum specific mappings of genes to $\mathrm{GO}$ terms as well as additional information on $\mathrm{GO}$ terms. Based on these two annotation databases, 3283 of 4294 genes and 2491 of 2903 proteins were associated with 614 GO terms. For each data set, a GO matrix with the same number of rows as the corresponding expression data set was created. The columns of the GO matrix hold data describing the gene/protein affiliation to a certain GO term. If a gene/protein is associated with that GO term, the strength of the affiliation is computed as the ratio between 1 and the total number of genes/proteins associated with the GO term. CIA and IBC use directly the computed GO matrix. GSVD performs a GSE analysis based on org.Pf.plasmo.db and GO. $d b$. In this way, we make sure that all three methods are applied to the same data sets. Additional file 1 contains the GO terms with their GO names used in this study.

Our study comprises the analysis of four data sets: mRNA abundance data, protein abundance data, a GO matrix of mRNAs and a GO matrix of proteins. mRNA and protein abundances were computed with different algorithms, requiring a columnar z-score normalization of each data set. The GO matrices were computed based on the number of genes/proteins belonging to a particular GO term, which resulted in equal ranges of the entries in the matrices. Afterwards, they were joined and z-transformed. Columns (GO terms) that included only entries equal to zero (none of the associated genes or proteins was measured in the data set) were deleted before the normalization. After deletion, $614 \mathrm{GO}$ terms were available for further analysis.

\section{$\mathrm{CIA}$}

CIA was introduced by Dolèdec and Chessel [30] as an extension of Tucker's inter-battery method [37] for the study of species-environment relationships of ecology data. CIA was applied to genome and agr groups data of S. aureus [38] and to physico-chemical properties of amino-acids and the amino-acid composition of E.coli proteins [39]. Culhane et al. [40] applied this method to two gene expression data sets, and Fagan et al. [1] used it as an integrative analysis method for gene and protein data.

CIA is a multivariate analysis method that identifies relationships between two data sets by maximizing the covariance between them. CIA starts by performing a multivariate analysis like principal component analysis (PCA) [41], non-symmetrical correspondence analysis (NSC) [42] or correspondence analysis (CA) [43] on each individual data set. The produced results are a set of principal axes that maximize the projected variability (inertia) of each data set independently. Each set of axes spans a new multidimensional gene and protein space. Based on the computed axes, CIA identifies one axis in each new multidimensional space on which the projected data sets present maximal covariance and simultaneously maximal standard deviations. Thereby, CIA maximizes the covariance between the two data sets. Global correlation or co-structure between the data sets is measured by the RV coefficient [44]. For mathematical details on CIA, please refer to [30]. CIA computation steps are summarized in a concise flowchart in Figure 1. CIA is available in the R packages made4 [45] and ade4 [46].

CIA has two major advantages: It can be applied to data sets with considerable more variables (genes and proteins) than samples (life cycle stages), and the variables in the two data sets do not have to match one another.

Additional information such as GO annotations can be superimposed on the CIA plots. This overlay was already done for CA [47], and is also possible for CIA [1]. GO term projections are obtained by first normalizing the two GO matrices in the same way as the expression data sets and then multiplying them by the weights of the genes/proteins resulting from the NSC, followed by CIA analyses. The projection scores computed in this way show GO term associated with the measured genes/ proteins in relation to the life cycle stages.

\section{GSVD}

The GSVD was developed as an extension of the singular value decomposition (SVD) that was already used directly as an analysis method $[48,49]$ and indirectly as part of a PCA [50,51]. In a study by Golub et al. [52], GSVD was used as a comparative analysis method [3] for two gene expression data sets of cell cycle data from yeast and humans.

GSVD is based on the joint decomposition of both data sets as shown in equations (1) and (2):

$$
\begin{aligned}
& G=U_{1} \Sigma_{1} X^{-1} \\
& P=U_{2} \Sigma_{2} X^{-1} .
\end{aligned}
$$

Matrices $G$ and $P$ contain the gene and protein abundance data. The rows of the common matrix $X^{-1}$ are named genelets. In [3] it was shown that these genelets can be regarded as processes captured by both data sets. The genelets are expressed only in the corresponding arraylets (columns of $U_{1}$ and $U_{2}$ ) with a relative significance measured by the generalized eigenvalues $\left(\sigma_{1, i}, \sigma_{2, i}\right)$ from the diagonals of $\Sigma_{1}$ and $\Sigma_{2}$. The relative significance of a genelet in the gene data set relative to the protein data set is measured by an antisymmetric angular distance calculated as shown in equation (3): 


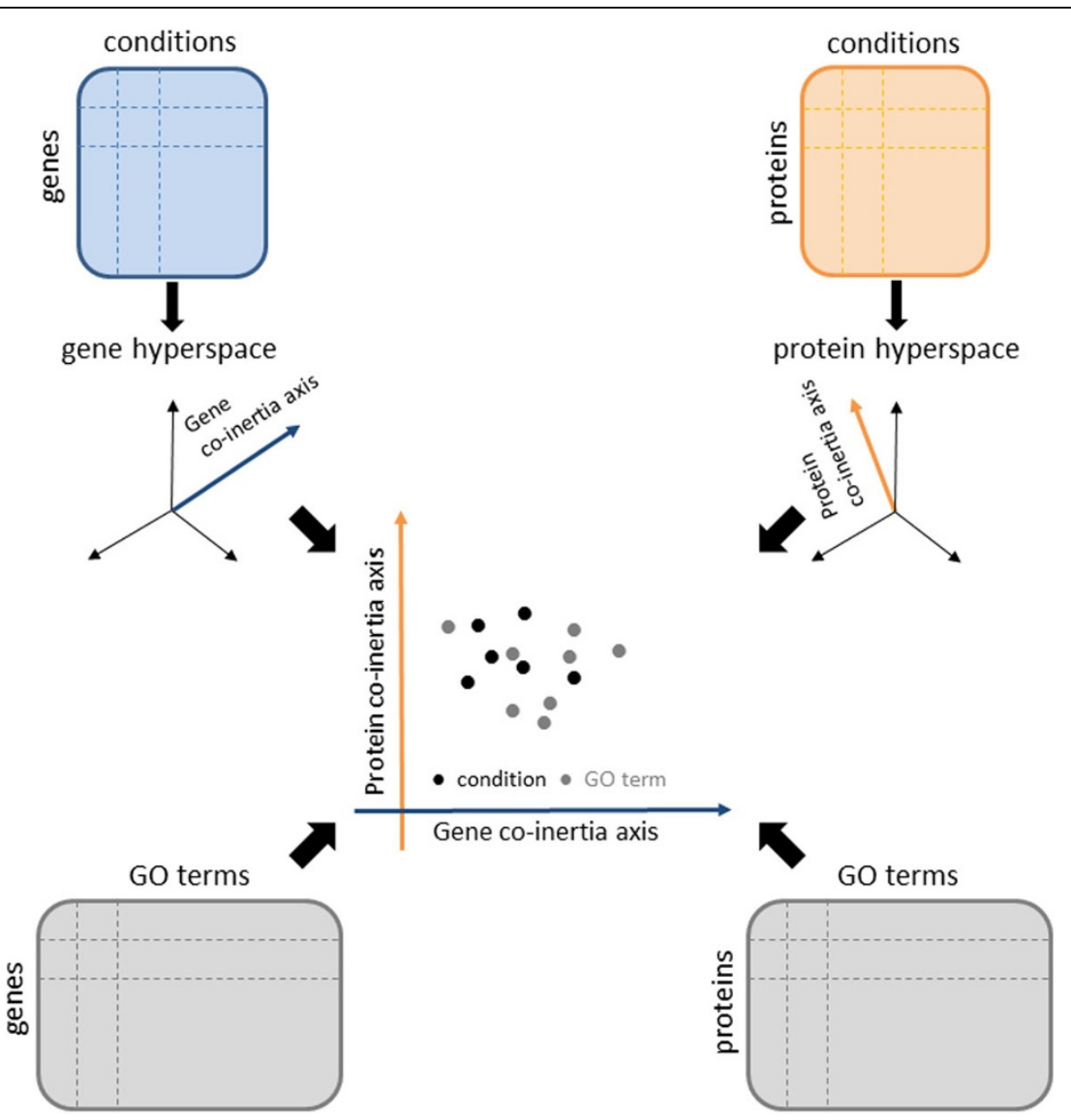

Figure 1 Flowchart of CIA. The gene expression matrix (blue) contains the genes in the rows and the conditions in the columns. The protein expression matrix (orange) contains the proteins in the rows and the conditions in the columns. The conditions in our study correspond to the life cycle stages of $P$. falciparum. Genes and proteins annotated to the considered GO terms are gathered in a separate matrix (gray). The gene and the protein expression matrices are transformed into a new hyperspace. The pair maximizing the covariance is computed from the axes spanning the two hyperspaces. These two axes (gene and proteins co-inertia axes) span a new space where the conditions (life cycle stages) can be plotted. Additionally, GO terms can be projected into the CIA space and visualized together with the life cycle stages. Figure adapted from [30].

$$
\theta_{i}=\arctan \left(\frac{\sigma_{1, i}}{\sigma_{2, i}}\right)-\frac{\pi}{4}
$$

An angular distance between $-\pi / 4$ and $-\pi / 8$ represents a high significance of the $i^{\text {th }}$ genelet in the second data set relative to the first data set. If the value of the angular distance ranges between $\pi / 8$ and $\pi / 4$, then the $i^{t h}$ genelet has a high significance in the first data set relative to the second data set. The $i^{\text {th }}$ genelet shows equal significance in both data sets if the angular distance ranges between $-\pi / 8$ and $\pi / 8$ (see equation (4)). In our study, the first matrix contains mRNA abundance data, the second matrix protein abundance data and significances are assigned as follows:

$$
\theta_{i} \in \begin{cases}{[-\pi / 4,-\pi / 8]} & \text { protein space } \\ {[-\pi / 8, \pi / 8]} & \text { gene and protein space (4) } \\ {[\pi / 8,-\pi / 4]} & \text { gene space. }\end{cases}
$$

A summary of the computation flow is shown in a block diagram in Figure 2.

Alter et al. [3] used a Mathematica implementation of a numerically robust GSVD algorithm based on [52,53], which we reimplemented in $\mathrm{R}$.

In order to discover the processes captured by the genelets, a restrictive gene set enrichment (GSE) analysis is performed on $50 \%$ of the genes and/or proteins showing the highest absolute values in the corresponding arraylets. The GSE analysis is performed with the R package GOstats [54], which computes the statistically significantly enriched GO terms based on the hypergeometrical distribution.

IBC

The basic idea of biclustering (co-clustering or two-way clustering) was presented in [55], but it took almost thirty years until the method was applied to gene expression data [56]. In the last two decades, biclustering has 


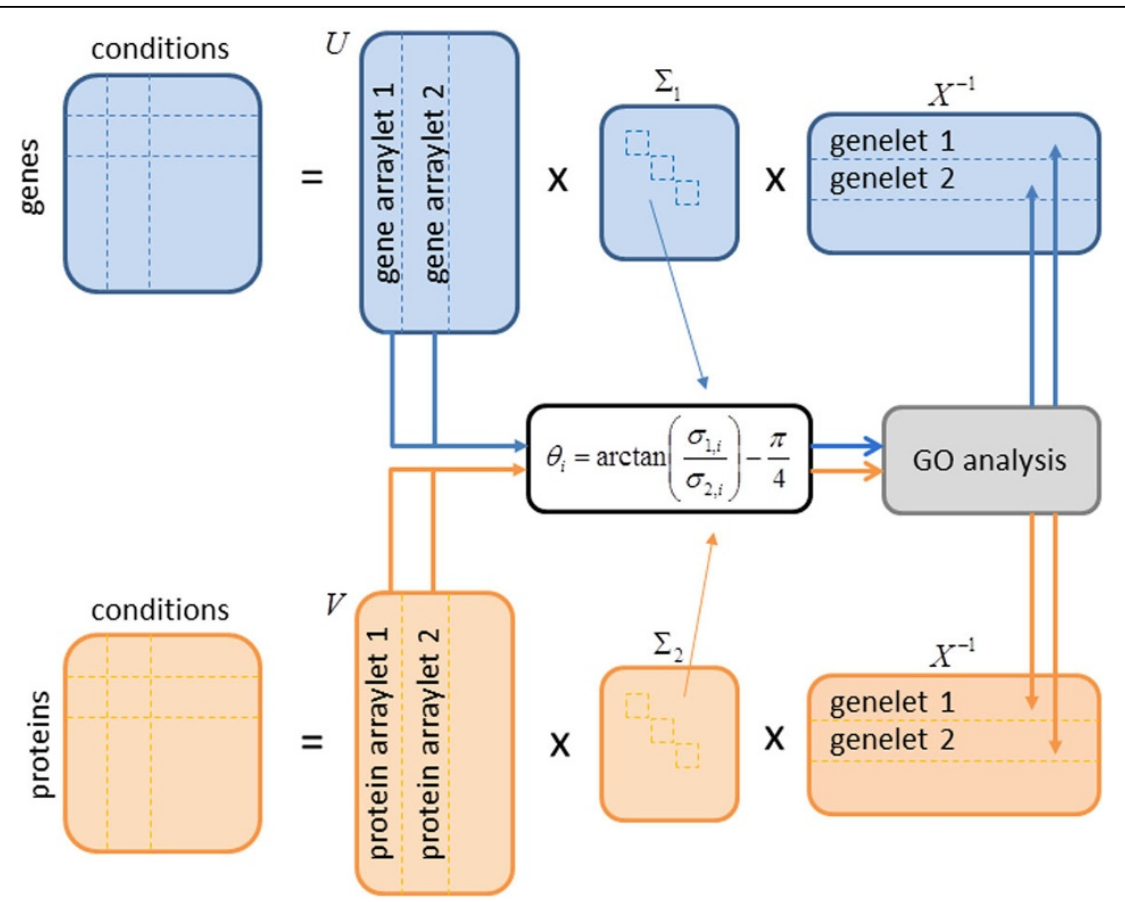

Figure 2 Flowchart of GSVD. The gene expression matrix (blue) contains the genes in the rows and the conditions in the columns. The protein expression matrix (orange) contains the proteins in the rows and the conditions in the columns. The conditions in our study correspond to the life cycle stages of $P$. falciparum. Genes and proteins annotated to the considered GO terms are gathered in a separate matrix (gray). The gene and the protein expression matrices are each decomposed in three matrices according to equations (1) and (2). The matrices $U$ and $V$ contain the arraylets, which encode for the expression of genes and proteins in the corresponding genelets $X^{1}$, which represent the cellular state in the measurement conditions. According to the angular distance $\theta_{i,}$ which is computed from the generalized eigenvalues $\sigma_{1, i}$ and $\sigma_{2, i}$, a restricted GSE analysis is performed on the genes and/or proteins with the absolute (from a mathematical point of view) highest values in the arraylets in order to assign GO terms to the corresponding genelets. Figure adapted from [3].

become more and more popular [57-59]. In contrast to clustering, where either rows or columns are clustered, biclustering performs clustering of rows and columns simultaneously. The members of the obtained biclusters are as similar to one another and as different from the other biclusters as possible. Figure 3 presents how mRNA abundances, protein expression and GO terms are assembled to a complete data set and how the resulting biclusters could look like.

There are four types of possible biclusters as reviewed in $[31,60,61]$. Biclusters can have (i) equal values over rows and columns as well as (ii) equal values over rows or columns. They can also have (iii) coherent values, which means that each column or row can be computed by adding or multiplying a constant to the previous column or row. The forth type of bicluster has (iv) coherent evolutions, which means that the exact value of a matrix entry is not important, but whether the values increase or decrease over rows or columns. The biclustering algorithm used here is included in the $\mathrm{R}$ package biclust [62].

The types of computed biclusters vary. There are single biclusters where only one bicluster is found in the whole data set as well as exclusive rows and/or exclusive columns biclusters. Non-overlapping and non-exclusive biclusters can also be computed. The fifth type is the arbitrarily positioned overlapping biclusters. Graphical representations of the different categories of biclusters can be found in [31].

Most of the biclustering algorithms implemented depend on the starting point of the search and thus may lead to different results in consecutive runs. Additionally, biclustering does not result in a perfect data separation, as overlapping biclusters are possible. As a remedy, the biclust package provides a robust method that delivers stable and reliable results. This function includes the repeated use of one algorithm in combination with several parameter settings and/or subsamples of the data. A modified version of the Jaccard index is used for the combination of the resulting biclusters, which in case of two biclusters takes into account the fraction of row-columns combinations in both biclusters to all rowcolumn combinations. For detailed mathematical definitions, please refer to [31].

Analogous to integrative clustering, we define integrative biclustering as the biclustering of two or more data sets. Integrative clustering was already applied to copy 


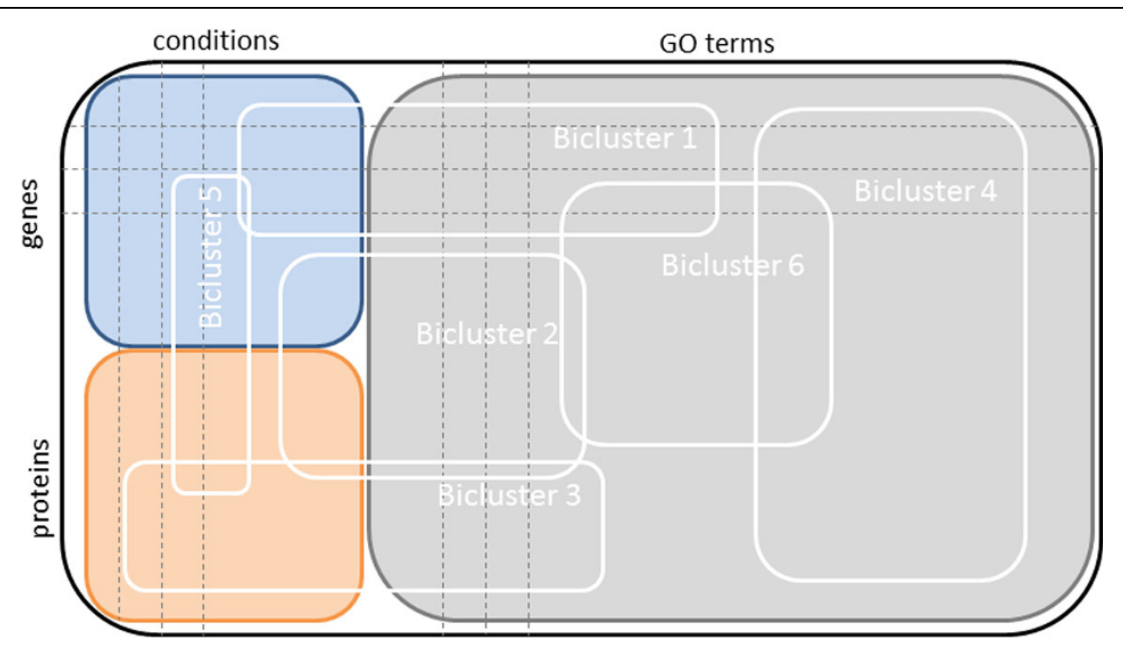

Figure 3 Block diagram of IBC. The gene expression matrix (blue) contains the genes in the rows and the conditions in the columns. The protein expression matrix (orange) contains the proteins in the rows and the conditions in the columns. The conditions in our study correspond to the life cycle stages of $P$. falciparum. Genes and proteins annotated to the considered GO terms are gathered in a separate matrix (gray). The three matrices are combined to a new matrix, which is subjected to biclustering. The resulting biclusters can include genes, proteins, conditions, GO terms or any combination of these.

number and gene expression data in order to identify novel breast tumours subgroups [63]. Mo and colleagues [5] describe integrative clustering of genomic, epigenomic and transcriptomic profiling.

Integrative biclustering was applied to gene expression, protein interaction, growth phenotype and transcription factor binding data in [64] in order to reveal modularity and organization in the yeast molecular network. We apply integrative biclustering to a matrix consisting of the mRNA and protein abundance data and of the corresponding GO matrices. The genes and the proteins are represented by rows, whereas the samples and the GO terms by columns. Before biclustering can be carried out, discretization is necessary. Here the built-in function discretize of the $\mathrm{R}$ package biclust [31] was used. After appropriate processing, the result of IBC was loaded into Cytoscape [65] to obtain a network view of the associations.

\section{Results and discussion}

Results of each analysis method can be divided into method-specific associations and general associations. The general associations are used to compute results common to all three methods.

\section{$\mathrm{ClA}$}

With CIA we visualize the six life cycle stages in the gene and protein space (Figure 4). We observe that the coinertia $\times$ axis separates the intraerythrocytic cycle stages (trophozoite, ring, schizont, merozoite) from gametocytes and sporozoites. In the erythrocytes, the cycle begins with the ring stage, followed by the trophozoite stage. Trophozoites mature into schizonts, which cause the rupture of blood cells resulting in the release of merozoites. In Figure 1, this exact sequence within the intraerythrocytic cycle can be observed. The sporozoites are the sexual stage of the mosquito and will be released in the blood stream of the infected organism. The ring stage can develop into a gametocyte and can be ingested by a mosquito. In addition to the life cycle stages, GO terms can also be represented through projections in the CIA plot (Figure 5).

A mapping between numbers and $\mathrm{GO}$ terms can be found in Additional file 1. Detailed representations of the division limits for the specific and general associations are shown in Additional file 4.

\section{General associations}

General associations resulting from CIA are distributed as follows: In gene space, GO terms in the first trigonometric quadrant are associated with trophozoites, GO terms in the second quadrant with gametocytes and GO terms in the third quadrant with sporozoites. GO terms in the first and forth quadrant, which were not identified as specific for trophozoits are associated with rings, schizonts and merozoits. Due to the proximity of stages in the CIA gene space, a more specific distribution to each stage is not possible. In protein space the associations are produced as follows: For gametocytes and sporozoites, we follow the same criteria as in gene space. For the distribution of GO terms to trophozoits, rings, schizonts and merozoits, we divide the first and forth quadrant in three sectors. GO terms that form angles of at least 30 degrees with the positive co-inertia $\times$ axis are associated with trophzoits. GO terms with an angle between -10 and 30 degrees are associated with rings and schizonts. 


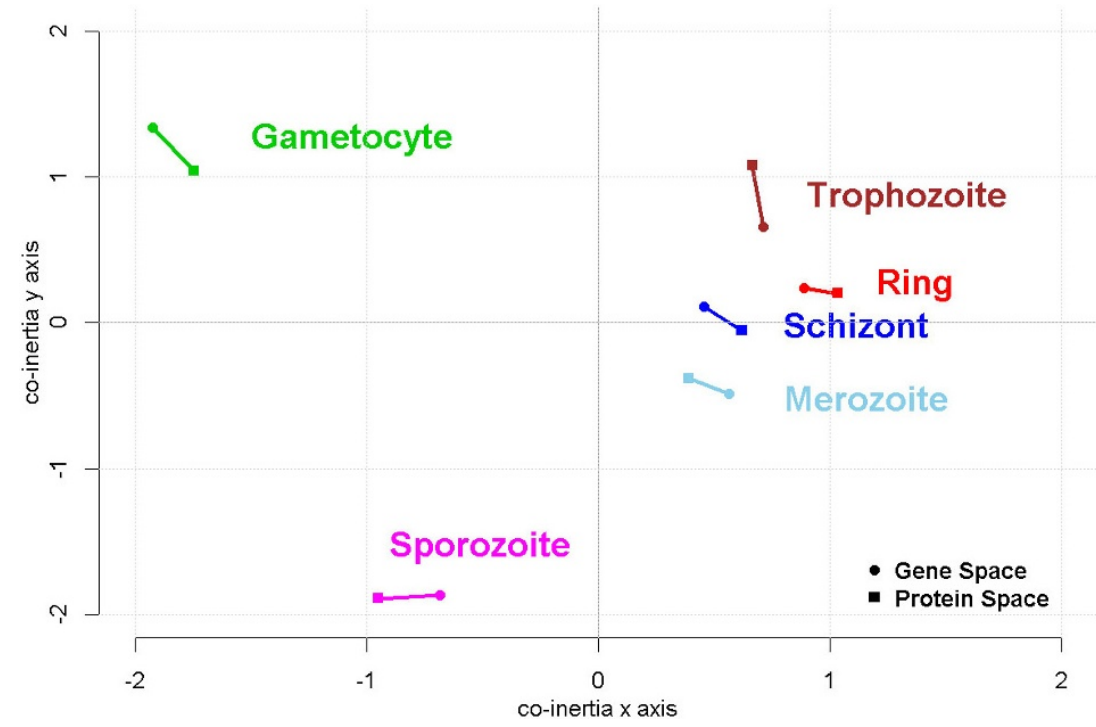

Figure 4 Co-inertia analysis - results. CIA offers the possibility to visualize the gene and protein space projections of the six life cycle stages of P. falciparum in one plot. The projection in gene space are represented by circles and in the protein space by squares. For each life cycle stage, the two corresponding projections are connected through a line. We observe that the y axis separates the intraerythrocytic cycle from the stages gametocyte and sporozoite.

GO terms with an angle wider than -10 degrees are associated with merozoits. Since GSVD and IBC discover associations only in common space (gene and protein space), the CIA associations for each life cycle are computed as the set union of the associations in gene and the associations in protein space. These general associations are shown in Additional files 2 and 3. The overlap between the general association in gene and protein space are shown in Figure 6.

\section{Method-specific associations}

In addition to the general results, method-specific associations of GO terms with life cycle stages are observed. For these associations, the direction of the projected GO terms is considered. From the general associations, we take those GO terms that have a distance of at least 0.1 to the origin of the coordinate systems. An exception is made for gametocytes in protein space. A threshold of 0.05 is more appropriate here due to the spacial

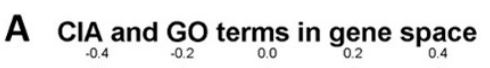

B CIA and GO.
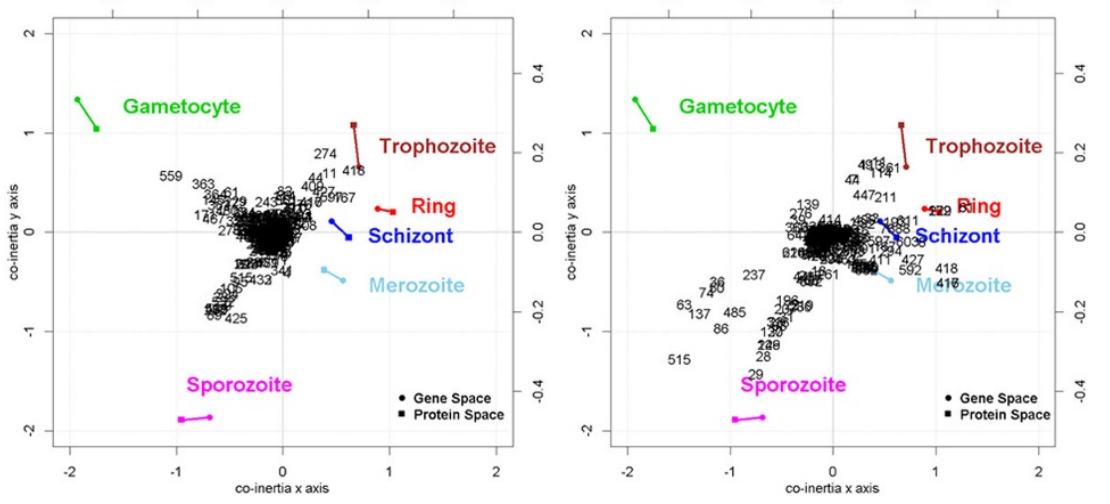

Figure 5 Co-inertia analysis and GO terms - results. In addition to the life cycle stages, GO terms can also be projected into the CIA plot. A) projections of the $\mathrm{GO}$ terms in gene space and B) projections of the $\mathrm{GO}$ terms in the protein space. Each $\mathrm{GO}$ term is represented by a number. Please note that the life cycle stages and the GO terms are plotted on different scales. The lower and left axes represent the life cycle stages and the upper and right axes represent for the GO terms. In gene space we observe a clear projection of the GO terms in the direction of gametocytes and sporozoites. In protein space, GO terms are projected clearly in direction of sporozoites and the intraerythrocytic cycle. 


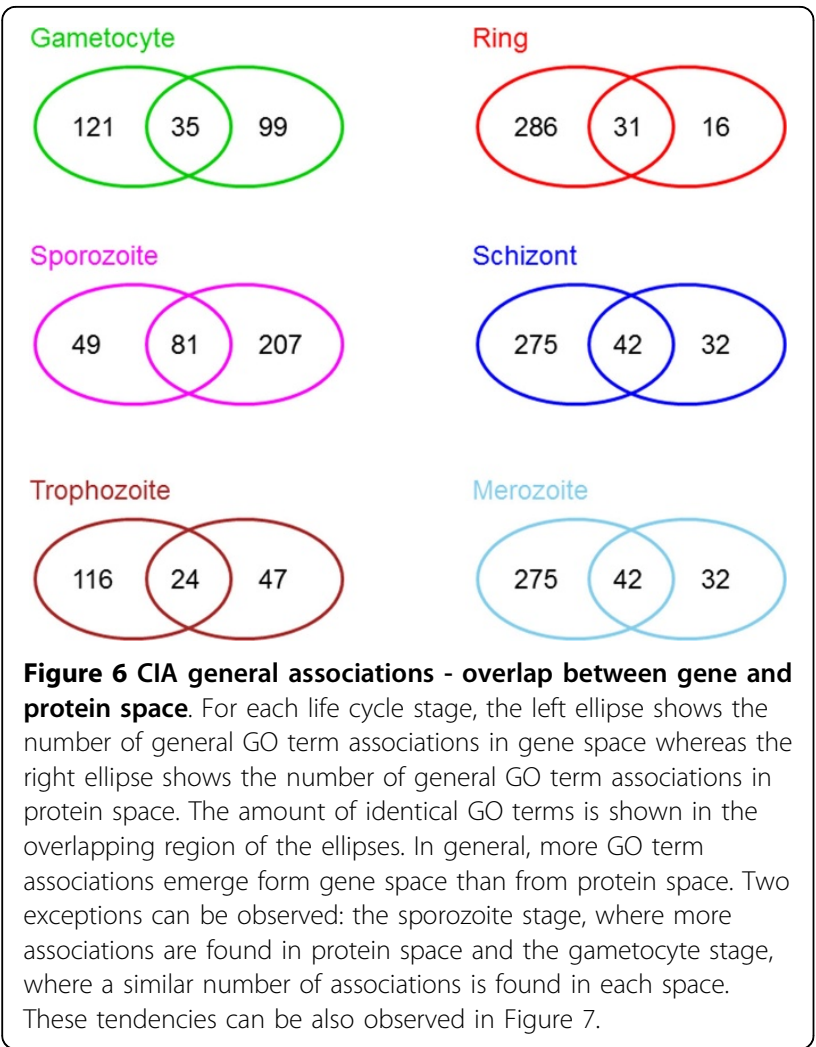

distribution of GO terms relative to the origin. These considerations result in GO term associations with gametocyte, trophozoite and sporozoite in gene space. Details are presented in Table 1 and Additional file 5. In the protein space, clear GO term associations with gametocyte, sporozoite, trophozoite and merozoite stages are found (Table 2 and Additional file 6). Additional file 6 also includes associations with the stages ring and schizont.

According to Figure 5, where for readability reasons GO terms are represented by numbers from 1 to 614, some of the most remarkable associations in gene space are: GO:0006071 glycerol metabolic process (559) and GO:0002720 positive regulation of cytokine production (363) for gametocytes; GO:0006101 citrate metabolic process (425) and GO:0016255 attachment of GPI anchor to protein (89) for sporozoites; GO:0006591 ornithine metabolic process (274) and GO:0006094 gluconeogenesis (418) for trophozoites. In protein space we observe: GO:0045454 cell redox homeostasis (139) and GO:0044262 cellular carbohydrate metabolic process (276) for gametocytes; GO:0006928 cellular component movement (551) and GO:0015991 ATP hydrolysis coupled proton transport (29) for sporozoites; GO:0006412 translation (11) and GO:0019538: protein metabolic process (361) for trophozoites; GO:0006334 nucleosome assembly (61) and GO:0050776 regulation of immune response (8) for ring and schizonts; GO:0042594 response to starvation (592), GO:0000045 autophagic vacuole assembly (416) and GO:0002253 activation of immune response (417) for merozoites.

The overlap between the projections in gene and protein space is modest. Three GO terms were projected in the direction of trophozoites in gene and in protein space: GO:0006412 translation (11), GO:0006414 translational elongation (44) and GO:0044257 cellular protein metabolic process (114).

\section{GSVD}

As the final step of the GSVD, a restrictive gene set enrichment analysis (GSE) is performed. The type of performed GSE analysis is based on the angular distance that encodes for each life cycle stage the significance of the gene set relative to the protein set. If the angular distances are between $-\frac{\pi}{8}$ and $\frac{\pi}{8}$, then the gene and protein data sets are of equal significance, and the GSE is conducted in the common space. The common space is defined by the gene and the protein data set. This is the case for all life cycle stages (Figure 7). If we compare the angular distance with zero, we obtain a separation of the intraerythrocytic cycle (angular distances bigger than zero) from other stages (angular distances smaller than zero). The restrictive GSE performs a GSE for each life cycle stage on $50 \%$ of the genes and proteins that present the highest absolute values in the corresponding arraylets.

\section{General associations}

All resulting GO terms having a p value smaller than 0.05 are considered to be general associations. These GO terms are shown in Additional file 7.

\section{Method-specific associations}

The method-specific GO terms are a subset of the general associations consisting of the top 15 GO terms, with the smallest $\mathrm{p}$ values. The method-specific associations are presented in Tables 3 and 4 and in Additional file 8. Biologically relevant associations include: GO:0051805/GO:0051807 evasion or tolerance if immune/defense response of other organism involved in symbiotic interaction, GO:0051832 avoidance or defenses of other organism involved in symbiotic interaction, and GO:0052173 response to defenses (immune response) of other organism involved in symbiotic interaction for trophozoites and schizonts. The other stages are associated with more general GO terms such as GO:0044237 cellular metabolic process, GO:0019538 protein metabolic process and GO:0046474 glycerophospholipid biosynthetic process.

\section{IBC}

The IBC results include two types of biclusters: (i) biclusters containing genes, proteins, GO terms and life 
Table 1 CIA specific GO term association in gene space to the gametocyte stage.

\begin{tabular}{ll}
\hline CIA: Gametocyte in gene space \\
\hline 61 & GO:0006334: nucleosome assembly \\
\hline 145 & GO:0006072: glycerol-3-phosphate metabolic process \\
\hline 361 & GO:0006465: signal peptide processing \\
\hline 363 & GO:0007131: reciprocal meiotic recombination \\
\hline 364 & GO:0002720: positive regulation of cytokine production involved in immune response \\
\hline 467 & GO:0006359: regulation of transcription from RNA polymerase III promoter \\
\hline 580 & GO:0051604: protein maturation \\
\hline 559 & GO:0001819: positive regulation of cytokine production \\
\hline
\end{tabular}

In this table GO term association in gene space to the life cycle stage gametocyte are presented. The numbers in the left column correspond to the numbers in graphic $\mathrm{A}$ of Figure 5

cycle conditions and (ii) biclusters containing genes, proteins and GO terms. Since we are interested in GO terms associations with life cycle stages, we will use only the first type of biclusters for further analysis. If a GO term is in the same bicluster as a life cycle stage, this GO term is associated with that life cycle stage. If there are more life cycle stages in a bicluster, the GO terms are associated with all these life cycle stages. If a life cycle stage is included in more that one bicluster, GO terms from all biclusters are associated with that life cycle stage. IBC discovered 20 biclusters and 9 of them contained life cycle stages and GO terms. A network view of the results is shown in Figure 8.

\section{General and method-specific associations}

Since a life cycle stage is either included in a bicluster or not and as a consequence is either associated to a GO term or not, it is not possible to distinguish between general and method-specific associations. Figure 8 shows a vast amount of genes (in orange), proteins (in light blue), GO terms (in yellow) and the six life cycle stages: gametocyte (in green), sporozoite (in pink), trophozoite (in brown), ring (in red), schizont (in dark blue) and merozoite (in light blue). The different biclusters resulting from the analysis can be identified through the colour of their

Table 2 CIA specific GO term association in protein space to the gametocyte stage.

\begin{tabular}{ll}
\hline CIA: Gametocyte in protein space \\
\hline 1 & GO:0009405: pathogenesis \\
\hline 39 & GO:0007165: signal transduction \\
\hline 64 & GO:0007155: cell adhesion \\
\hline 139 & GO:0045454: cell redox homeostasis \\
\hline 276 & GO:0044262: cellular carbohydrate metabolic process \\
\hline 366 & GO:0006103: 2-oxoglutarate metabolic process \\
\hline
\end{tabular}

In this table GO term association in protein space to the life cycle stage gametocyte are presented. The numbers in the left column correspond to the numbers in graphic B of Figure 5 edges. The exact associations with the life cycle stages are shown in Additional file 9.

\section{Common results}

In this section, we present $\mathrm{GO}$ associations observed in all three methods. The common associations are shown in Figure 9. These associations are based on gene as well as protein information and are therefore considered to be in the common space. The GO associations computed with $\mathrm{R}$ were converted into a compatible format and loaded into Cytoscape. We observe here that the gametocytes are linked to the rest of the network through only one general GO term, GO:0009987 cellular process. The sporozoite stage is also loosely connected to the network through two GO terms, GO:0009056 catabolic process and GO:0009116 nucleoside metabolic process. The intraerythrocytic cycle, composed of trophozite, ring, schizont and merozoite are highly interconnected. The merozoite stage presents a high number of associations with specific GO terms such as GO:0030260 entry into host cell and GO:0044409 entry into host. Trophozoites are associated with a small number of GO terms, including GO:0050896 response to stimulus, GO:0006096 glycolysis, GO:0006006 glucose metabolic process and GO:0006091 generation of precursor metabolites and energy. The stages schizont and ring are connected through the GO terms GO:0006955 immune response, GO:0050776 regulation of immune response, GO:0006325 chromatin organization and GO:0006091 generation of precursor metabolites and energy. It is also interesting to see that merozoits and schizonts are linked only through the GO term GO:0009116 nucleoside metabolic process.

\section{Relative proportions of common and methods-specific} results

In the case of CIA, one can observe a high overlap between the common results and the CIA specific GO terms associations: $8 \mathrm{GO}$ terms (GO:0005975 carbohydrate metabolic process, GO:0006644 phospholipid metabolic process, GO:0008654 phospholipid biosynthetic process, GO:0045017 glycerolipid biosynthetic process, 


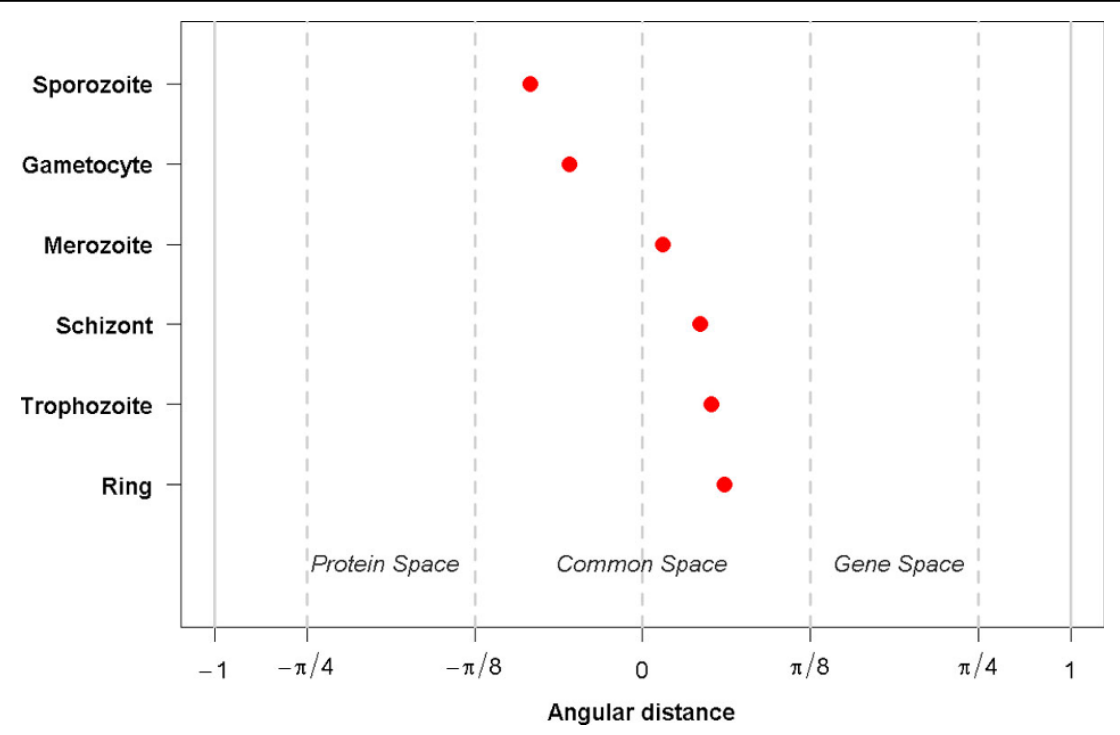

Figure 7 Generalized singular value decomposition - angular distances. GSVD computes angular distances between gene and protein space. In general, the angular distances map to the common space, for which restricted GSE analysis is performed on the gene and on the proteins arraylets. Nevertheless, while angular distances belonging to the intraerythrocytic cycle stages have positive values and show a tendency to the gene space, the angular distances of gametocytes and sporozoites have negative values and thus a tendency towards protein space. These preferences are also reflected by the amount of GO term associations emerging from the gene and from the protein space (see also Figure 6).

GO:0006661 phosphatidylinositol biosynthetic process, GO:0046488 phosphatidylinositol metabolic process, GO:0006506 GPI anchor biosynthetic process, GO:001 6255 attachment of GPI anchor to protein) associated by CIA with merozoites in protein space, 8 GO terms (GO:0 009058 biosynthetic process, GO:0051276 chromosome

Table 3 GSVD specific GO term association to gametocyte stage in common space.

\begin{tabular}{ll}
\hline GSVD: Gametocyte in common space \\
\hline GO:0044238 & primary metabolic process \\
\hline GO:0008152 & metabolic process \\
\hline GO:0044237 & cellular metabolic process \\
\hline GO:0045017 & glycerolipid biosynthetic process \\
\hline GO:0043170 & macromolecule metabolic process \\
\hline GO:0034645 & cellular macromolecule biosynthetic process \\
\hline GO:0046474 & glycerophospholipid biosynthetic process \\
\hline GO:0009059 & macromolecule biosynthetic process \\
\hline GO:0022613 & ribonucleoprotein complex biogenesis \\
\hline GO:0044260 & cellular macromolecule metabolic process \\
\hline GO:0019538 & protein metabolic process \\
\hline GO:0046486 & glycerolipid metabolic process \\
\hline GO:0042254 & ribosome biogenesis \\
\hline GO:0006839 & mitochondrial transport \\
\hline GO:0009987 & cellular process \\
\hline
\end{tabular}

In this table GSVD based GO term association in common space to the life cycle stage gametocyte are presented. organization, GO:0006325 chromatin organization, GO:0050776 regulation of immune response, GO:0006955 immune response, GO:0006096 glycolysis, GO:0006334 nucleosome assembly, GO:0044237 cellular metabolic process) associated by CIA with rings and schizonts in protein space and 3 GO terms (GO:0009117 nucleotide metabolic process, GO:0006163 purine nucleotide metabolic process, GO:0009116 nucleoside metabolic process) associated by CIA with sporozoites in protein space. Only two GO terms (GO:0006096 glycolysis and GO:000 6006 glucose metabolic process associated with trophozoites) from gene space, coincide with GO terms from the common results. Protein activity characteristics derived from CIA show considerable similarities to the other two methods.

Six specific results of GSVD for the life cycle stage ring coincide with the common GO terms associations with this stage (GO:0009058 biosynthetic process, GO:0019538 protein metabolic process, GO:0044237 cellular metabolic process, GO:0008152 metabolic process, GO:0055114 oxidationreduction process, GO:0006091 generation of precursor metabolites and energy). There are three identical associations for the stage merozoite (GO:0019538 protein metabolic process, GO:0016311 dephosphorylation and GO:0006470 protein dephosphorylation). For each of the other stages, only one GO term from the common associations coincides with the method-specific associations (GO:0009987 cellular process for gametocytes, GO:0006091 generation of precursor metabolites and energy for trophozoites, GO:0020033 antigenic 
Table 4 GSVD specific GO term association to trophozoite stage in common space.

\begin{tabular}{ll}
\hline GSVD: Trophozoite in common space \\
\hline GO:0044403 & symbiosis, encompassing mutualism through parasitism \\
\hline GO:0044419 0051704 & interspecies interaction between organisms \\
\hline GO:0009607 & multi-organism process \\
\hline GO:0006952 & response to biotic stimulus \\
\hline GO:0051707 & defense response \\
\hline GO:0051805 & response to other organism \\
\hline GO:0051807 GO:0051832 & evasion or tolerance of immune response of other organism involved in symbiotic interaction \\
\hline GO:0051834 & evasion or tolerance of defense response of other organism involved in symbiotic interaction \\
\hline GO:0052173 & avoidance of defenses of other organism involved in symbiotic interaction \\
\hline GO:0052564 & evasion or tolerance of defenses of other organism involved in symbiotic interaction \\
\hline GO:0020033 & response to defenses of other organism involved in symbiotic interaction \\
\hline GO:0051809 & response to immune response of other organism involved in symbiotic interaction \\
\hline GO:0006091 & antigenic variation \\
\hline
\end{tabular}

In this table GSVD based GO term association in common space to the life cycle stage trophozoite are presented.

variation for schizonts and GO:0009056 catabolic process for sporozoites). In conclusion, the ring stage is very well characterized by the GSVD, which is almost in complete agreement with the other methods. The properties of the other stages do not coincide with the common results but

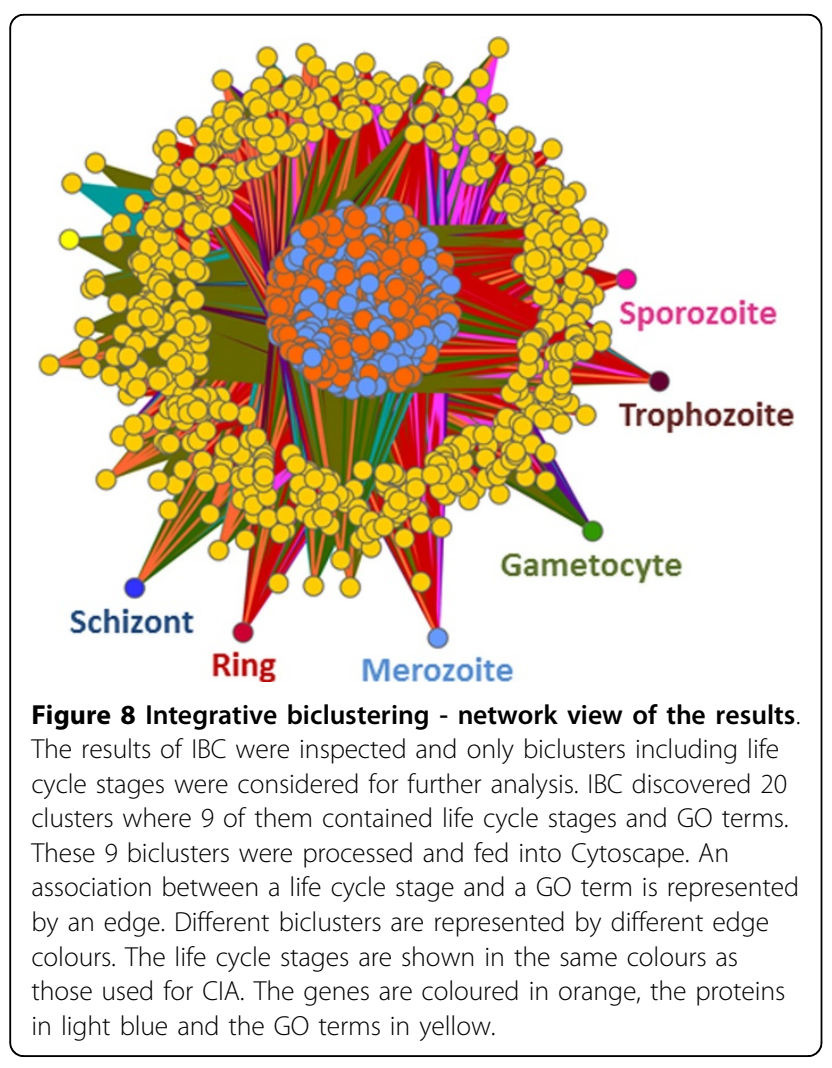

should definitely be considered for further analysis as they are highly significant.

In this study, we have applied three integrative analysis methods to a data set containing mRNA and protein abundances from the six life cycle stages of P. falciparum. The use of integrative analysis methods allows to consider all annotated and measured genes (3283) and proteins (2491), not limited by the 2230 pairs of genes and proteins as when it was first published in [9]. The integration of knowledge on different levels allows the linking of the data sets based on samples and not on variables (genes, protein).

We presented three different integrative analysis methods, each with its own justification: CIA discovers biological processes on the basis of maximal covariance. GSVD decomposes the data sets into genelets and arraylets and conducts a modified GSE analysis on them. IBC computes biclusters according to the distance between genes, proteins and GO terms.

We have shown method-specific results as well as results common to all three analysis methods. In the case of CIA, the associations in protein space presented a high overlap with the common results. This was not the case for the associations in gene space. In case of the sporozoite stage, GSVD associations are very simmilar to the common results. For the other stages, GSVD yielded different mappings compared to the common results. As a GO term is associated or not with a life cycle stage, only general but no method-specific results were computed for IBC.

For CIA, it is important to consider that GO term associations are done through projection, whereas GSVD maps GO terms to individual stages through restricted GSE analysis and IBC assigns GO terms to life cycle stages 


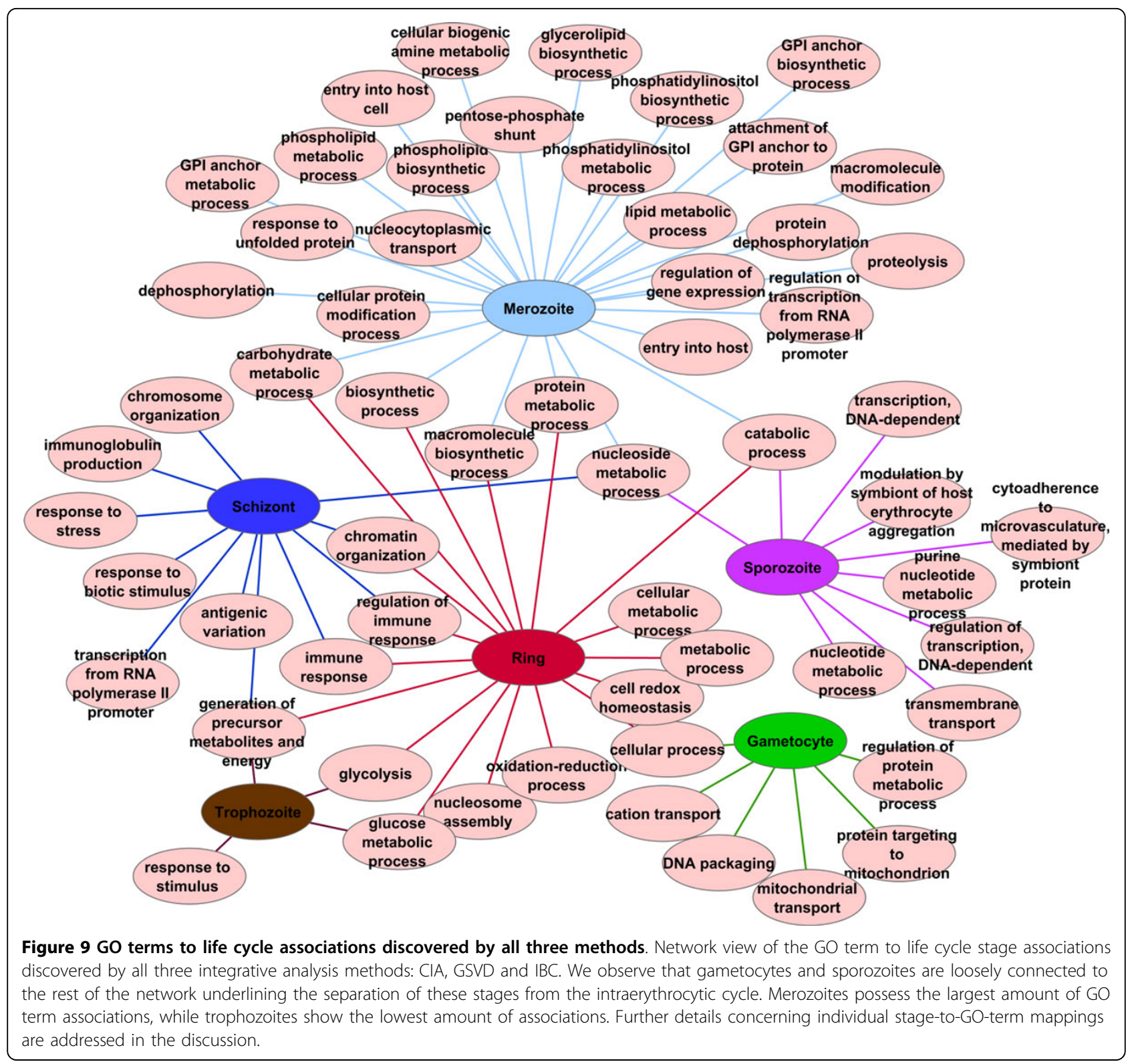

through the distance to the corresponding life cycle stage. Another important aspect is that with CIA it is not possible to associate one $\mathrm{GO}$ term to more than one life cycle stage, while this is possible with GSVD and IBC. Due to the heterogeneous computational methods, we proposed taking the intersect of the three obtained results.

In the three-fold validated network view of the biological processes (Figure 9), we observe the separation of the intraerytrocytic cycle (merozite, ring, trophozoite and schizont) from sporozoites and gametocytes. While the stages of the intraerytrocytic cycle are tightly connected to one another, sporozoites share two biological processes and gametocytes share only one biological process with the rest. Gametocytes and sporozoites do not possess any common processes, reflecting the differences between these stages. Gametocytes are released into the blood stream, from where they travel to the liver, while sporozoites represent the sexual stage and lie dormant in cell cycle arrest until ingestion by a mosquito.

The data used here was gathered in order to investigate the role of post-transcriptional regulation in P. falciparum [9]. For this, only pairs of mRNA and the corresponding protein were considered, resulting in the exploitation of $89 \%$ of the proteins and $60 \%$ of the genes that were experimentally measured. By employing integrative analysis methods we were able to take all measured data into account. 
LeRoch and coworkers [9] mention that there is a "bias in proteomic analysis of whole-cell lysates, in that such methods may fail to detect secreted or membrane proteins present in low abundance" such as GPI anchors. Due to the integrative approach, our analysis associates several GO terms related to GPI anchors proteins (GO:0006506 GPI anchor biosynthetic process, GO:0016255 attachment of GPI anchor to protein, GO:0006661 phosphatidylinositol biosynthetic process, GO:0046488 phosphatidylinositol metabolic process) with the merozoite stage, prevailing over this shortcoming. These associations are in agreement with [66], where distinct protein classes, with a focus on merozoite surface antigens, are discussed. The importance of GPI anchor proteins in the merozoite stage is well known and very important in immune evasion $[67,68]$.

Other biological processes mentioned in [9] such as glycolysis and cell invasion, without any life cycle mapping, were also found in our network: GO:0044409 entry into host and GO:0030260 entry into host cell, both associated with the merozoite stage. Our network assigns GO:0006096 glycolysis to the stage trophozoite, in concordance to [69] where the transcriptome of P. falciparum was characterized.

Simmilar to our findings, cell invasion was associated with merozoites in [67], where a proteomic view of the $P$. falciparum life cycle was presented. Other concordances with [67] include the assignment of GO:0006508 proteolysis to the merozoite stage. During trophozoite stage, digestion of haemoglobin takes place. Our network maps GO:0006091 generation of precursor metabolites and energy to trophozoites, confirming the importance of energy production during this stage. As mentioned by Florens et al. [67], sporozoites are injected into the blood stream where they have to survive in a hostile environment. Based on our combined results, sporozoites are associated with GO:0020013 modulation by symbiont of host erythrocyte aggregation and GO:0020035 cytoadherence to microvasculature, mediated by symbiont protein, which reflects the process of survival. Additionally, sporozoites are associated with metabolism and transcription, as was shown in Figure 5 of [67]. Our results reflect these findings by mapping GO:0006163 purine nucleotide metabolic process, GO:0009117 nucleotide metabolic process, GO:0006351 transcription, DNA dependent and GO:0006355 regulation of transcription, DNA dependent to the sporozoite stage.

During gametocyte stage, DNA processing and energy production is highly regulated, as mentioned in [67]. In agreement, our results assign GO:0006323 DNA packaging, GO:0006839 mitochondrial transport and GO:0006626 protein targeting to mitochondrion to the gametocytes.

The analysis of the $P$. falciparum proteome by LaCount and colleagues [70] associated the intraerythrocytic cycle with chromatin modification, transcriptional regulation, mRNA stability/processing, ubiquitination, nucleic acid metabolism and invasion of host cells. Since our analysis corresponds to individual life cycle stages, we can associate biological processes to a certain stage of the intraerythrocytic cycle, providing a more detailed description of P. falciparum. According to our findings, chromatin modification takes place during schizont stage (GO:0006325 chromatin organization, GO:0051276 chromosome organization); merozoites are associated with GO:0006357 regulation of transcription from RNA polymerase II promotor and schizonts with GO:0042795 transcription from RNA polymerase II promotor; merozoites are associated with GO:0009116 nucleoside metabolic process; invasion of host cells can be observed during merozoite stage (GO:0044409 entry into host and GO:0030260 entry into host cell). Ubiquitination was only detected through its parent term GO:0044267 cellular protein metabolic process, which was associated with merozoites.

Fagan et al. [1] conducted CIA on a slightly different data set which took $P$. berghei orthologues into account and showed that GO:0006412 biosynthesis is associated to the intraerythrocytic cycle. In our network, several more specialized biosynthetic processes are associated with the merozoite stage: GO:0009059 macromolecule biosynthetic process, GO:0008654 phospholipid biosynthetic process, GO:0045017 glycerolipid biosynthetic process, GO:0006661 phosphatidylinositol biosynthetic process, GO:0006506 GPI anchor biosynthetic process, as well as the GO term GO:0006412 biosynthetic process itself.

The importance of immune evasion through antigenic variation was highlighted by Winzeler [71]. Our results show that this process is related to the schizont stage, as our analysis associates GO:0020033 antigenic variation, GO:0006955 immune response, GO:0050776 regulation of immune response, GO:0002377 immunoglobulin production, GO:0006950 response to stress and GO:0009607 response to biotic stimulus with this stage.

The role of lipids during merozoite stage was already shown in 1988 by Mikkelsen et al. [72]. Our computed network associates merozoites with GO:0006644 phospholipid metabolic process, GO:0008654 phospholipid biosynthetic process, GO:0046486 glycerolipid metabolic process and GO:0006629 lipid metabolic process, reflecting this early finding.

Phosphorilation and dephosphoryliation processes play an important role in the internalization step of meroziotes [73], a fact that is also reflected by our results. Merozoites are associated with GO:0016311 dephosphorylation and GO:0006470 protein dephosphorylation.

The role of the pentose phosphate pathway in P. falciparum was disscused in [74], without a clear life cycle stage assignment. Our computed network view maps GO:0006098 pentose-phosphate shunt to merozoites. 
As shown in [75], REDOX complexes play an important role during ring stage, which is in agreement with our results that associate ring stage with GO:0045454 cell redox homeostasis and GO:0055114 oxidation-reduction process.

Roth [76] showed that carbohydrate metabolism is a key metabolic process connecting the host cells with P. falciparum. Our findings assign GO:0005975 carbohydrate metabolic process to merozoite and ring stages.

Most of our network associations are in concordance with several publications dealing with the characterization of $P$. falciparum, based on transcriptome $[68,69]$ and proteome $[67,70]$ characterization data. A considerable amount of the findings in the above publications are concentrated in our results of the used integrative analysis methods. Our findings are more detailed through the association with a specific life cycle stage rather than, e.g. the whole intraerythrocytic cycle as well as through the association of a child GO term instead of a parent GO term to the corresponding stage. Our study unifies individual findings from several publications of the past 25 years of research. Not all results from the publications mentioned above are present in our network. This could be due to the fact that none of the cited publications, except [9], used the same data sets as we did. Llinas et al. [68] compared the three P. falciparum strains 3D7, Dd2 and HB3 through the measurement of the gene expression profiles of 6287,5294 and 6415 genes during the intraerythrocytic cycle. Bozdech et al. [69] considered in their analysis of the intraerythrocytic cycle transcriptome the expression of 5508 genes. LaCount and colleagues [70] analysed 1267 proteins for their protein interaction network of $P$. falciparum. In [67], Florens et al. use approximately 2400 proteins in order to create a proteomic view of the $P$. falciparum life cycle. The other studies are based on lab experiments on smaller groups of genes or proteins [66,71-73,75].

Additionally, our combined network view of life cycle stage dependent GO term association provides a new overview for the vaccine research and offers new insight in the interdependencies between life cycle stages. Possibly it could even identify key biological processes on which vaccine researchers could concentrate their work.

\section{Conclusion}

In this study we have shown the power of integrative analysis methods. We presented three very different approaches that showed significant overlap of results. We compared our findings against the past 25 years of $P$. falciparum research and showed that the obtained network unifies, on the life cycle level, results from analyses done separately on transcriptome and proteome data, as well as results from the lab, which were performed on small groups of genes or proteins. Further investigations are needed to obtain a complete map of the biological processes activated during the life cycle of $P$. falciparum. Measurement of the transcriptome and proteome of $P$. falciparum, exploiting the advantages of current high throughput technologies, would complement the spectrum of biological process presented here. An increase of our understanding of $P$. falciparum could be achieved by performing the integrative analysis methods on the molecular function and/or cellular compartment level of gene ontology. Further work could also cover the identfication of genes and proteins that play key roles during the life cycle of $P$. falciparum through integrative analysis on gene and protein level, not only on GO term level.

\section{Additional material}

Additional file 1: GO term mapping. PDF file containing the mapping between the numbers used in the CIA plots, GO terms and GO names. Additional file 2: CIA general GO term associations in gene space. PDF file containing the CIA general GO term associations in gene space.

Additional file 3: CIA general GO term associations in gene space. PDF file containing the CIA general GO term associations in protein space.

Additional file 4: CIA division limits. PDF file containing the CIA division limits for general (left) and specific (right) associations. The colours of the areas correspond to the colours of the stages they are associated with.

Additional file 5: CIA specific GO term associations in gene space. PDF file containing the CIA specific GO term associations in gene space. Additional file 6: $\mathrm{CIA}$ specific GO term associations in protein space. PDF file containing the CIA specific GO term associations in protein space.

Additional file 7: GSVD general GO term associations. PDF file containing the GSVD based general associations of GO terms to life cycle stages in common space.

Additional file 8: GSVD specific GO term associations. PDF file containing the GSVD based specific associations of GO terms to life cycle stages in common space.

Additional file 9: IBC general GO term associations. PDF file containing the IBC based general associations of GO terms to life cycle stages in common space.

\section{Competing interests}

The authors declare that they have no competing interests.

Authors' contributions

GGT and DM conceived the original idea. OAT performed the analysis, summarized and compared the results and drafted the manuscript under the supervision of GGT. All the authors have read and approved the final manuscript.

\section{Acknowledgements}

This work has been supported by the Federal Ministry of Economy, Family and Youth (BMWFJ), the Federal Ministry of Traffic, Innovation and Technology (bmvit), the Styrian Business Promotion Agency SFG, the Standortagentur Tirol and ZIT - Technology Agency of the City of Vienna through the COMET-Funding Program managed by the Austrian Research Promotion Agency FFG [COMET K2 Grant 824186, Austrian Centre of Industrial Biotechnology]. We thank Orly Alter for providing the Mathematica code of the GSVD and Julia Feichtinger for critically reading the manuscript. 


\section{Declarations}

This article has been published as part of BMC Systems Biology Volume 8 Supplement 2, 2014: Selected articles from the High-Throughput Omics and Data Integration Workshop. The full contents of the supplement are available online at http://www.biomedcentral.com/bmcsystbiol/supplements/8/S2. The publication costs for this article were funded by COST-BMBS, Action BM1006 "Next Generation Sequencing Data Analysis Network", SeqAhead and by the Austrian Centre of Industrial Biotechnology

\section{Authors' details}

${ }^{1}$ Core Facility Bioinformatics, Austrian Centre of Industrial Biotechnology, Graz, Austria. ${ }^{2}$ Institute of Genomics and Bioinformatics, Graz University of Technology, Graz, Austria. ${ }^{3}$ Cell Design and Engineering, Austrian Centre of Industrial Biotechnology, Vienna, Austria. ${ }^{4}$ Department of Biotechnology, BOKU-VIBT University of Natural Resources and Life Sciences, Vienna, Austria. ${ }^{5}$ Omics Center Graz, Graz, Austria.

Published: 13 March 2014

\section{References}

1. Fagan A, Culhane AC, Higgins DG: A multivariate analysis approach to the integration of proteomic and gene expression data. Proteomics 2007, 7:2162-2171.

2. Lazar C, Taminau J, Meganck S, Steenhoff D, Coletta A, Weiss Solis DY, Molter C, Duque R, Bersini H, Nowe A: GENESHIFT: a Non-Parametric Approach for Integrating Microarray Gene Expression Data Based on the Inner Product as a Distance Measure Between the Distributions of Genes. IEEE/ACM Trans Comput Biol Bioinf 2013, 2:383-292.

3. Alter $\mathrm{O}$, Brown $\mathrm{PO}$, Botstein D: Generalized singular value decomposition for comparative analysis of genome-scale expression data sets of two different organisms. Proc Natl Acad Sci USA 2003, 100:3351-3356.

4. Wang KS, Liu X: Integrative Analysis of Genome-wide Expression and Methylation Data. J Biom Biostat 2013, 4:4-6.

5. Mo Q, Wang S, Seshan VE, Olshen AB, Schultz N, Sander C, Powers RS, Ladanyi M, Shen R: Pattern discovery and cancer gene identification in integrated cancer genomic data. Proc Natl Acad Sci USA 2013, 110:4245-4250.

6. Kockmann T, Gerstung M, Schlumpf T, Xhinzhou Z, Hess D, Beerenwinkel N, Beisel C, Paro R: The BET protein FSH functionally interacts with ASH1 to orchestrate global gene activity in Drosophila. Genome Biol 2013, 14:R18.

7. Chen Z, Zhang W: Integrative Analysis Using Module-Guided Random Forests Reveals Correlated Genetic Factors Related to Mouse Weight. PLoS Comput Biol 2013, 9:e1002956.

8. Gerstein MB, Kundaje A, Hariharan M, Landt SG, Yan KK, Cheng C, Mu XJ, Khurana E, Rozowsky J, Alexander R, Min R, Alves P, Abyzov A, Addleman N, Bhardwaj N, Boyle AP, Cayting P, Charos A, Chen DZ, Cheng Y, Clarke D, Eastman C, Euskirchen G, Frietze S, Fu Y, Gertz J, Grubert F, Harmanci A, Jain P, Kasowski M, Lacroute P, Leng J, Lian J, Monahan H, O'Geen H, Ouyang Z, Partridge EC, Patacsil D, Pauli F, Raha D, Ramirez L, Reddy TE, Reed B, Shi M, Slifer T, Wang J, Wu L, Yang X, Yip KY, Zilberman-Schapira G, Batzoglou S, Sidow A, Farnham PJ, Myers RM, Weissman SM, Snyder M: Architecture of the human regulatory network derived from ENCODE data. Nature 2012, 489:91-100.

9. Le Roch KG, Johnson JR, Florens L, Zhou Y, Santrosyan A, Grainger M, Yan SF, Williamson KC, Holder A, Carucci DJ, Yates JR, Winzeler E: Global analysis of transcript and protein levels across the Plasmodium falciparum life cycle. Genome Res 2004, 14:2308-2318.

10. Cox B, Kislinger T, Emili A: Integrating gene and protein expression data: pattern analysis and profile mining. Methods 2005, 35:303-314.

11. Cagney G, Park S, Chung C, Tong B, Dushlaine CO, Shields DC, Emili A: Human Tissue Profiling with Multidimensional Protein Identification Technology. J Proteome Res 2005, 4:1757-1767.

12. Corbin RW, Paliy O, Yang F, Shabanowitz J, Platt M, Lyons CE, Root K, McAuliffe J, Jordan MI, Kustu S, Soupene E, Hunt DF: Toward a protein profile of Escherichia coli: comparison to its transcription profile. Proc Natl Acad Sci USA 2003, 100(16):9232-9237.

13. Chen $\mathrm{Yr}$, Juan Hf, Huang Hc, Huang Hh, Lee Yj, Liao My, Tseng CW, Lin LI, Chen Jy, Wang Mj, Chen Jh, Chen Yj: Quantitative Proteomic and Genomic Profiling Reveals Metastasis-Related Protein Expressio Patterns in Gastric Cancer Cells research articles. J Proteome Res 2006, 5:2727-2742.
14. Griffin TJ: Complementary Profiling of Gene Expression at the Transcriptome and Proteome Levels in Saccharomyces cerevisiae. Mol Cell Proteomics 2002, 1:323-333.

15. Mootha VK, Bunkenborg J, Olsen JV, Hjerrild M, Wisniewski JR, Stahl E, Bolouri MS, Ray HN, Sihag S, Kamal M, Patterson N, Lander ES, Mann M: Integrated Analysis of Protein Composition, Tissue Diversity, and Gene Regulation in Mouse Mitochondria. Cell 2003, 115:629-640.

16. Washburn MP, Koller A, Oshiro G, Ulaszek RR, Plouffe D, Deciu C, Winzeler E, Yates JR: Protein pathway and complex clustering of correlated mRNA and protein expression analyses in Saccharomyces cerevisiae. Proc Natl Acad Sci USA 2003, 100:3107-3112.

17. Kislinger T, Cox B, Kannan A, Chung C, Hu P, Ignatchenko A, Scott MS, Gramolini AO, Morris Q, Hallett MT, Rossant J, Hughes TR, Frey B, Emili A: Global Survey of Organ and Organelle Protein Expression in Mouse: Combined Proteomic and Transcriptomic Profiling. Cell 2006, 125:173-186.

18. Nie L, Wu G, Brockman FJ, Zhang W: Integrated analysis of transcriptomic and proteomic data of Desulfovibrio vulgaris: zero-inflated Poisson regression models to predict abundance of undetected proteins. Bioinformatics 2006, 22:1641-1647.

19. Haider S, Pal R: Integrated Analysis of Transcriptomic and Proteomic Data. Curr Genomics 2013, 14:91-110.

20. Hwang D, Smith JJ, Leslie DM, Weston AD, Rust AG, Ramsey S, de Atauri P, Siegel AF, Bolouri H, Aitchison JD, Hood L: A data integration methodology for systems biology: Experimental verification. Proc Nat/ Acad Sci USA 2005, 102:17302-17307.

21. Hwang D, Rust AG, Ramsey S, Smith JJ, Leslie DM, Weston AD, de Atauri P, Aitchison JD, Hood L, Siegel AF, Bolouri H: A data integration methodology for systems biology. Proc Natl Acad Sci USA 2005, 102:17296-17301.

22. Hahne H, Mäder U, Otto A, Bonn F, Steil L, Bremer E, Hecker M, Becher D: A comprehensive proteomics and transcriptomics analysis of Bacillus subtilis salt stress adaptation. J Bacteriol 2010, 192:870-882.

23. Verhoef S, Ballerstedt $H$, Volkers RJM, de Winde JH, Ruijssenaars HJ: Comparative transcriptomics and proteomics of $p$-hydroxybenzoate producing Pseudomonas putida S12: novel responses and implications for strain improvement. Appl Microbiol Biotechnol 2010, 87:679-690.

24. Takemasa I, Kittaka N, Hitora T, Watanabe M, Matsuo El, Mizushima T, Ikeda M, Yamamoto H, Sekimoto M, Nishimura O, Doki Y, Mori M: Potential biological insights revealed by an integrated assessment of proteomic and transcriptomic data in human colorectal cancer. Int J Oncol 2012, 40:551-559.

25. Piruzian E, Bruskin S, Ishkin A, Abdeev R, Moshkovskii S, Melnik S, Nikolsky Y, Nikolskaya T: Integrated network analysis of transcriptomic and proteomic data in psoriasis. BMC Syst Biol 2010, 4:41-53.

26. Perco P, Mühlberger I, Mayer G, Oberbauer R, Lukas A, Mayer B: Linking transcriptomics and proteomic data on the level of protein interaction networks. Electrophoresis 2010, 31:1780-1789.

27. Joyce $A R$, Palsson BO: The model organism as a system: integrating 'omics' data sets. Nat Rev Mol Cell Biol 2006, 7:198-210.

28. Hecker M, Lambeck S, Toepfer S, van Someren E, Guthke R: Gene regulatory network inference: data integration in dynamic models-a review. BioSystems 2009, 96:86-103.

29. Zhang $W, L i F$, Nie L: Integrating multiple 'omics' analysis for microbial biology: application and methodologies. Microbiology 2010, 156:287-301.

30. Dolèdec S, Chessel D: Co-inertia analysis: an alternative method for studying species-environment relationships. Freshw Biol 1994, 31:277-294.

31. Kaiser S: Biclustering: Methods, Software and Application. PhD thesis Ludwig-Maximilians-University Munich, Department of Statistics; 2011.

32. Ashburner M, Ball CA, Blake JA, Botstein D, Butler H, Cherry JM, Davis AP, Dolinski K, Dwight SS, Eppig JT, Harris MA, Hill DP, Issel-tarver L, Kasarskis A, Lewis S, Matese JC, Richardson JE, Rubin GM, Sherlock G: Gene Ontology: tool for the unification of biology. Nat Genet 2000, 25:25-29.

33. Le Roch KG, Zhou Y, Blair PL, Grainger M, Moch JK, Haynes JD, De La Vega P, Holder A, Batalov S, Carucci DJ, Winzeler E: Discovery of gene function by expression profiling of the malaria parasite life cycle. Science 2003, 301:1503-1508

34. R Development Core Team: R: A Language and Environment for Statistical Computing R Foundation for Statistical Computing, Vienna, Austria; 2013 [http://www.R-project.org], [ISBN 3-900051-07-0].

35. Carlson M: org.Pf.plasmo.db: Genome wide annotation for Malaria , [R package version 2.8.1] 
36. Carlson M: GO.db: A set of annotation maps describing the entire Gene Ontology, [R package version 2.8.0].

37. Tucker LR: An inter-battery method for factor analysis. Psychometrika 1958, 23:111-136.

38. Jarraud S, Mougel C, Thioulouse J, Lina G, Meugnier H, Forey F, Etienne J, Vandenesch F, Jarraud S, Mougel C, Thioulouse J, Lina G, Nesme X, Etienne J: Relationships between Staphylococcus aureus Genetic Background, Virulence Factors, agr Groups (Alleles), and Human Disease. Infect Immun 2002, 70:631-641.

39. Thioulouse J, Lobry J: Co-inertia analysis of amino-acid physico-chemical properties and protein composition with the ADE package. Comput App/ Biosci 1995, 11:321-329.

40. Culhane AC, Perrière G, Higgins DG: Cross-platform comparison and visualisation of gene expression data using co-inertia analysis. $B M C$ Bioinformatics 2003, 4:59.

41. Jolliffe IT: Principal Component Analysis New York Berlin Heidelberg: Springer-Verlag; 2002.

42. Gimaret-Carpentier C, Chessel D, Pascal J: Non-symmetric correspondence analysis: an alternative for species occurrences data. Plant Ecol 1998, 138:97-112.

43. Greenacre M: Theory and Applications of Correspondence Analysis London: Academic Press; 1983.

44. Robert P, Escoufier Y: A Unifying Tool for Linear Multivariate Statistical Methods: The RV-Coefficient. App/ Statist 1976, 25:257-265

45. Culhane AC, Thioulouse J, Perrière G, Higgins DG: MADE4: an R package for multivariate analysis of gene expression data. Bioinformatics 2005, 21:2789-2790.

46. Chessel D, Dufour AB, Thioulouse J: The ade4 package - I: One table methods. $R$ News 2004, 5:5-10.

47. Busold CH, Winter S, Hauser N, Bauer A, Dippon J, Hoheisel JD, Fellenberg K: Integration of GO annotations in Correspondence Analysis: facilitating the interpretation of microarray data. Bioinformatics 2005, 21:2424-2429.

48. Alter O, Brown PO, Botstein D: Singular value decomposition for genomewide expression data processing and modeling. Proc Natl Acad Sci USA 2000, 97:10101-10106.

49. Nielsen TO, West RB, Linn SC, Alter O, Knowling MA, Connell JXO, Zhu S, Fero M, Sherlock G, Pollack JR, Brown PO, Botstein D, Rijn MVD: Mechanisms of disease Molecular characterisation of soft tissue tumours: a gene expression study. The Lancet 2002, 359:1301-1307.

50. Wen X, Fuhrman S, Michaels GS, Carr DB, Smith S, Barker JL, Somogy R: Large-scale temporal gene expression mapping of central nevous system development. Proc Natl Acad Sci USA 1998, 95:334-339.

51. Hilsenbeck SG, William E, Schiff R, Connell O, Hansen RK, Osborne K, Fuqua SAW: Statistical Analysis of Array Expression Data as Applied to the Problem of Tamoxifen Resistance. J Natl Cancer Inst 1999, 91:453-459.

52. Golub GH, Van Loan CF: Matrix Computation Baltimore and London: Johns Hopkins University Press; 1996.

53. Paige CC, Saunders MA: Towards a Generalized Singular Value Decomposition. SIAM J Number Anal 1981, 18:398-405.

54. Falcon S, Gentleman R: Using GOstats to test gene lists for GO term association. Bioinformatics 2007, 23:257-258.

55. Hartigan JA: Direct Clustering of a Data Matrix. J Am Stat Assoc 1972 67:123-129.

56. Cheng Y, Church M: Biclustering of Expression Data. Proc Int Conf Intell Syst Mol Biol 2000, 8:93-103.

57. Getz G, Levine $E$, Domany E: Coupled two-way clustering analysis of gene microarray data. Proc Natl Acad Sci USA 2000, 97:12079-12084.

58. Ben-Dor A, Chor B, Karp R, Yukhini Z: Descovering local structure in gene expression data: The order preserving submatrix problem. J Comput Biol 2003, 10:373-384.

59. Murali T, Kasif S: Extracting conserved gene expression motifs from gene expression data. Pac Symp Biocomput 2003, 8:77-88.

60. Madeira SC, Oliveira AL: Biclustering algorithms for biological data analysis: a survey. IEEE/ACM Trans Comput Biol Bioinf 2004, 1:24-45.

61. Prelić A, Bleuler S, Zimmermann P, Wille A, Bühlmann P, Gruissem W, Hennig L, Thiele L, Zitzler E: A systematic comparison and evaluation of biclustering methods for gene expression data. Bioinformatics 2006, 22:1122-1129.
62. Kaiser S, Santamaria R, Tatsiana, Khamiakova, Sill M, Theron R, Quintales L, Leisch F: biclust: BiCluster Algorithms 2013 [http://CRAN.R-project.org/ package=biclust], [R package version 1.0.2].

63. Curtis C, Shah SP, Chin SF, Turashvili G, Rueda OM, Dunning MJ, Speed D, Lynch AG, Samarajiwa S, Yuan Y, Gräf S, Ha G, Haffari G, Bashashati A, Russell R, McKinney S, Langerød A, Green A, Provenzano E, Wishart G, Pinder S, Watson P, Markowetz F, Murphy L, Ellis I, Purushotham A, Børresen-Dale AL, Brenton JD, Tavaré S, Caldas C, Aparicio S: The genomic and transcriptomic architecture of 2,000 breast tumours reveals novel subgroups. Nature 2012, 486:346-352.

64. Tanay A, Sharan R, Kupiec M, Shamir R: Revealing modularity and organization in the yeast molecular network by integrated analysis of highly heterogeneous genomewide data. Proc Natl Acad Sci USA 2004, 101:2981-2986

65. Smoot M, Ono K, Ruscheinski J, Wang PL, Ideker T: Cytoscape 2.8: new features for data integration and network visualization. Bioinformatics 2011, 27:431-432.

66. Sanders PR, Gilson PR, Cantin GT, Greenbaum DC, Nebl T, Carucci DJ, McConville MJ, Schofield L, Hodder AN, Yates JR, Crabb BS: Distinct protein classes including novel merozoite surface antigens in Raftlike membranes of Plasmodium falciparum. J Biol Chem 2005, 280:40169-40176.

67. Florens L, Washburn MP, Raine JD, Anthony RM, Grainger M, Haynes JD, Moch JK, Muster N, Sacci JB, Tabb DL, Witney Aa, Wolters D, Wu Y, Gardner MJ, Holder Aa, Sinden RE, Yates JR, Carucci DJ: A proteomic view of the Plasmodium falciparum life cycle. Nature 2002, 419:520-526.

68. Llinás M, Bozdech Z, Wong ED, Adai AT, DeRisi JL: Comparative whole genome transcriptome analysis of three Plasmodium falciparum strains. Nucleic Acids Res 2006, 34:1166-1173.

69. Bozdech Z, Llinás M, Pulliam BL, Wong ED, Zhu J, DeRisi JL: The transcriptome of the intraerythrocytic developmental cycle of Plasmodium falciparum. PLOS Biol 2003, 1:E5.

70. LaCount DJ, Vignali M, Chettier R, Phansalkar A, Bell R, Hesselberth JR, Schoenfeld LW, Ota I, Sahasrabudhe S, Kurschner C, Fields S, Hughes RE: A protein interaction network of the malaria parasite Plasmodium falciparum. Nature 2005, 438:103-107.

71. Winzeler EA: Malaria research in the post-genomic era. Nature 2008, 455:751-756.

72. Mikkelsen RB, Kamber M, Wadwa KS, Lin PS, Schmidt-Ullrich R: The role of lipids in Plasmodium falciparum invasion of erythrocytes: a coordinated biochemical and microscopic analysis. Proc Natl Acad Sci USA 1988, 85:5956-5960.

73. Ward G, Fujioka H, Aikawa M, Miller L: Staurosporine Inhibits Invasion of Erythrocytes by Malarial Merozoites. Exp Parasitol 1994, 79:480-487.

74. Bozdech Z, Ginsburg H: Data mining of the transcriptome of Plasmodium falciparum: the pentose phosphate pathway and ancillary processes. Malaria J 2005, 4:17.

75. Mok S, Imwong M, Mackinnon MJ, Sim J, Ramadoss R, Yi P, Mayxay M, Chotivanich K, Liong KY, Russell B, Socheat D, Newton PN, Day NPJ, White NJ, Preiser PR, Nosten F, Dondorp AM, Bozdech Z: Artemisinin resistance in Plasmodium falciparum is associated with an altered temporal pattern of transcription. BMC Genomics 2011, 12:391.

76. Roth EJ: Plasmodium falciparum carbohydrate metabolism: a connection between host cell and parasite. Blood Cells 1990, 16:453-466.

doi:10.1186/1752-0509-8-S2-S4

Cite this article as: Tomescu et al:: Integrative omics analysis. A study based on Plasmodium falciparum mRNA and protein data. BMC Systems Biology 2014 8(Suppl 2):S4. 